\title{
Experimental and Numerical Correlation of Gravity Sag In Solar Sail Quality Membranes
}

\author{
Jonathan T. Black ${ }^{\square}$ \\ University of Kentucky, Lexington, KY 40506 \\ Jack Leifer ${ }^{2}$ \\ University of Kentucky, Paducah, KY 42002 \\ Joshua A. DeMoss ${ }^{3}$ \\ Virginia Polytechnic Institute, Blacksburg, VA 24061 \\ Eric N. Walker ${ }^{4}$ \\ George Washington University, NASA Langley Research Center, Hampton, VA 23681 \\ and \\ W. Keith Belvin ${ }^{5}$ \\ NASA Langley Research Center, Hampton, VA 23681
}

\begin{abstract}
Solar sails are among the most studied members of the ultra-lightweight and inflatable (Gossamer) space structures family due to their potential to provide propellentless propulsion. They are comprised of ultra-thin membrane panels that, to date, have proven very difficult to experimentally characterize and numerically model due to their reflectivity and flexibility, and the effects of gravity sag and air damping. Numerical models must be correlated with experimental measurements of sub-scale solar sails to verify that the models can be scaled up to represent full-sized solar sails. In this paper, the surface shapes of five horizontally supported $25 \mu \mathrm{m}$ thick aluminized Kapton membranes were measured to a 1.0 $\mathrm{mm}$ resolution using photogrammetry. Several simple numerical models closely match the experimental data, proving the ability of finite element simulations to predict actual behavior of solar sails.
\end{abstract}

\section{Introduction}

Qolar sails are part of the class of ultra-lightweight and inflatable gossamer space structures. They are

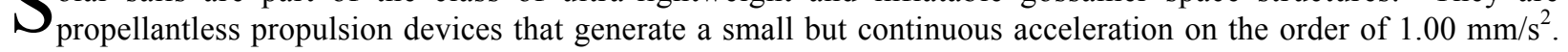
In order to achieve this acceleration rate, solar sails must be fabricated using membranes less than $5.0 \mu \mathrm{m}$ thick with areal densities less than $7.0 \mathrm{~g} / \mathrm{m}^{2}$. This constant acceleration will allow travel in non-Keplerian orbits, which are uniquely suited for several tasks such as polar observing and holding relatively stationary positions. Consequently solar sails have been proposed for use on several NASA missions planned for launch this decade. ${ }^{2,3}$

The thrust provided by a solar sail is generated when photons of sunlight are reflected by their specular surfaces, transferring momentum that propels the spacecraft. This momentum transfer, called solar pressure, is quite small $-9.12 \times 10^{-6} \mathrm{~N} / \mathrm{m}^{2}$ at $1 \mathrm{AU}-$ and requires that the sails be simultaneously very large and very lightweight to generate useful acceleration. It is impossible to effectively test full-scale models of square solar sails $100 \mathrm{~m}$ or more on a side on Earth due to vacuum chamber size limitations and an inability to compensate for the effects of gravity. Consequently, highly accurate numerical models will be used in place of experimental data. These models must be

\footnotetext{
${ }^{\square}$ Graduate Research Assistant, Department of Mechanical Engineering, AIAA Student Member.

${ }^{2}$ Assistant Professor, Department of Mechanical Engineering, AIAA Member

${ }^{3}$ Co-Op Student, Department of Mechanical Engineering, AIAA Student Member

${ }^{4}$ Graduate Research Assistant, Structural Dynamics Branch, AIAA Student Member

${ }^{5}$ Lead, Ultra-lightweight and Inflatable Structures Group, AIAA Senior Member
} 
correlated with laboratory sub-scale models, then altered to eliminate the influences of gravity and air damping and scaled up to reflect the full size of the sails.

As an initial step in the correlation process, several solar sail quality membranes were suspended horizontally with two different boundary support configurations. The static shape of each membrane was measured using a full-field, non-contact technique called dot projection photogrammetry to avoid extraneous loads introduced by more traditional sensors typically attached to the test article. These data are compared to numerical models with identical boundary and suspension conditions.

\section{Experimental Setup}

Several aluminum frames, including the one shown in Figure 1, were bolted to a steel backstop approximately 2.8 meters above the ground. The protruding corners of the frame were supported by steel wires also connected to the backstop. This extra support was provided to eliminate as much bending of the frame as possible, and to ensure that the test article was approximately horizontal. Each membrane was attached to the frame either along the entire perimeter (fixed-edge) or only at the corners (corner-supported) creating two different boundary support conditions. A total of five membranes were measured: square and triangular membranes, each with either corner-supported or fixed-edge conditions; and a circular membrane with a fixed-edge condition. The area behind (above) the membranes was open, allowing them to sag and wrinkle without obstruction from the frame.

Dot-projection photogrammetry was used to statically characterize the out-of-plane surface shape of each suspended membranes. Photogrammetry is the science of making precise shape measurements from photographs. To generate these measurements, multiple images taken simultaneously were loaded into the photogrammetry software and associated with the appropriate calibration parameters. These parameters were calculated independent of the measurement and allowed the software to remove distortions in the images caused by lens curvature and imperfections, focus, aperture setting, and other camera factors and aberrations.

The projector setup shown in Figure 2 created a grid of targets on each of the membranes. Four professional digital cameras imaged these targets. The photographs were taken simultaneously to minimize the possibility of membrane movement between images. After the images were loaded into the photogrammetry software and assigned the calibration parameters, each of the projected targets was marked to sub-pixel accuracy using an automatic least squares matching algorithm. The points, corresponding to the exact centers of the targets, were then matched across the images and a bundle adjustment algorithm was run that simultaneously iterated on the camera and point locations, all in three-dimensional space. The final result of the process was a set of threedimensional points that were exported for measurement ${ }^{8-10}$.

All photogrammetry measurements are initially un-scaled, meaning a scale and orientation were defined in post-processing. Invar scale bars (Figures 1 and 2), with targets exactly $1.1176 \mathrm{~m}$ (44.0 in) apart were used to scale the project. Because the retro-reflective targets on the scale bar required a flash for illumination, three additional images were taken after the measurement was completed, and referenced back to the four simultaneous images using projected and attached targets on the frame.

\section{Experimental Results}

The results shown here were generated from a contour mapping software using the five three-dimensional sets of points generated by the photogrammetry measurements. The software uses one of several different algorithms, selected by the user depending on the desired amount of smoothing and interpolation, to create "continuous" grids of regularly spaced points from the discrete data. A surface was then fit through each grid to produce the contour maps shown in Figures 3 through 7.

Figure 3 shows the surface profile of the first suspended test article, the square $25 \mu \mathrm{m}$ thick aluminized Kapton membrane one meter on a side. The membrane was attached to the frame along its perimeter (fixed-edge). Figure 3(a) is a shaded relief map of the membrane that shows the measured wrinkle pattern. The pattern runs diagonally to the right, probably the result of inaccuracies in the attachment of the membrane to the frame by hand. The rotated prospective provided by Figure 3(b) allows for quantitative evaluation of the amplitude of the wrinkles. The average amplitude of surface wrinkles is approximately $2 \mathrm{~mm}$, with smaller features clearly visible. Figure 4 shows the surface profile for the same type of square aluminized Kapton membrane, attached to the frame only at its corners, in the corner-supported boundary condition. As expected, a large sag of approximately $16 \mathrm{~mm}$ in amplitude is visible, as well as some smaller wrinkles running from the corners to the center of the membrane.

Figure 5 shows the surface profile of a right triangular aluminized Kapton membrane, $1.00 \mathrm{~m} \mathrm{X} 1.00 \mathrm{~m} \mathrm{X}$ $1.41 \mathrm{~m}$, attached to the frame along its perimeter. Figure 5(a) is a shaded relief map of the membrane that shows the measured wrinkle pattern. Figure 5(b) allows for determination of the wrinkle amplitude, which, at less than $1 \mathrm{~mm}$ 
in size, is less than that obtained for the similarly-mounted square membrane. Figure 6 shows the surface profile of the same type of right triangular aluminized Kapton membrane, attached to the frame at its corners. As expected, a large sag in the membrane approximately $14 \mathrm{~mm}$ in depth was observed.

Figure 7 shows the surface profile of the one meter diameter circular membrane attached to the frame along its perimeter. The wrinkle pattern of the membrane is visible in the shaded relief map shown in Figure 7(a), and the wrinkle amplitude read from Figure 7(b) averages approximately $2 \mathrm{~mm}$. The prospective provided by Figure 7(b) indicates slight frame misalignments.

These results show that successful non-contact measurement of winkle patterns less than one millimeter in amplitude is possible using dot-projection photogrammetry. The results are used as a standard against which results from the numerical models are compared to demonstrate their ability to accurately predict experimental results using fairly simple simulations.

\section{Numerical Models}

Numerical models were created for most of the membrane configurations and boundary conditions measured experimentally. The square, triangular, and circular $25 \mu \mathrm{m}$ thick aluminized Kapton membranes were suspended horizontally to simplify the modeling of gravity and to reflect the true loading condition of actual solar sails. While solar pressure is six orders of magnitude smaller than the force of gravity, it will be incident roughly normal to the surface of the sail. ${ }^{4}$ Due to the inaccuracies inherent in experimental data, repetitive load conditions, and processing and convergence limitations in the numerical simulations, the triangular membrane was only modeled in the corner-supported configuration and the circular membrane was not modeled.

Three finite element models were created using four node, six degrees of freedom per node shell elements and the following material properties for Kapton Type $100 \mathrm{Hn}$ Film: Young's Modulus $=2.5 \mathrm{GPa}$, Poisson's Ratio $=$ 0.34 , Density $=1420 \mathrm{~kg} / \mathrm{m}^{3}$ (Figures $8-10$ ) . $^{5-7}$ Figure 8 shows the square corner-supported configuration $1.0 \mathrm{~m}$ on each side with $0.002 \mathrm{~m} 45^{\circ}$ chamfer at each corner, reflecting the actual dimensions of the membranes shown in Figures 3 and 4. Figure 9 shows the square fixed-edge configuration with identical dimensions to the model in Figure 8. Figure 10 shows the corner-supported $1.0 \mathrm{~m}$ right triangular configuration with $0.002 \mathrm{~m} 45^{\circ}$ chamfer at the right angle and $0.001 \mathrm{~m} 45^{\circ}$ chamfer at the other two corners.

The gravity sags apparent in Figures 8 and 10 were induced using element sizes of 0.010 and $0.015 \mathrm{~m}^{2}$ respectively, symmetry, and a static, large deformation non-linear analysis solved using the arc-length method. ${ }^{6}$ The arc-length method applied gravity in 239 sub-steps, iteratively varying the applied load at each equilibrium convergence calculation within each sub-step to maintain total model convergence. The wrinkle pattern seen in Figure 9 was generated by adding an additional load step to the gravity step in which the top and bottom edges of the membrane were sheared $0.5 \mathrm{~mm}$ in opposite directions. This additional load step was solved using the nonlinear arc-length method discussed above with a number of load steps determined automatically. Many attempts were made to propagate the resulting wrinkles throughout the entire membrane by tensioning various edges in various directions in additional load steps without success. Figure 9 does, however, demonstrate the ability of this numerical method to produce steady state wrinkles similar in direction and amplitude to those seen in the experimental data using a fairly simple simulation that did not require the complex and detailed modeling of the contraction caused by the drying glue that was used to secure the membranes to the frames or other boundary intricacies. Despite the intentional simplicity of the models, a minimum of five hours of computer processor time was required to generate the results. More complex models would obviously produce more accurate results, but these simple models, run on standard desk-top computers, produced data of a level of fidelity that do not justify the added time, effort, and expense required by more complicated models.

\section{Correlation}

An examination of Figures 4 and 8 reveals good correlation between the experimental results and the numerical simulation. The numerical result in Figure 8 shows a gravity sag of $12.5 \mathrm{~mm}$, while the experimental data show a sag of approximately $16 \mathrm{~mm}$. The two figures also have similar, diamond-shaped global deflection patterns and similar, small-amplitude wrinkles running from the corners to the centers of the membranes. Figures 3 and 9 both show the fixed-edge boundary condition for the square membrane. While the wrinkles in Figure 9 do not propagate through the entire membranes as those in Figure 3, they show the same diagonal tendencies and have similar amplitudes of between 2 and $5 \mathrm{~mm}$. Figures 6 and 10 show high correlation in the corner-supported triangular membrane configuration. Both figures reveal a $14.0 \mathrm{~mm}$ deflection caused by gravity and similar deflection patters. The good to excellent correlation of the numerical models to the experimental data shows that simple finite element simulations can be used to approximate the actual behavior of solar sails and other gossamer

$-3-$ 
structures, allowing more detailed finite element models to be validated and scaled up to accurately predict the behavior of full-scale structures.

\section{Conclusions}

The correlation of numerical models with experimental data is an important step in the future development of solar sails. As an initial step in the process, small square, triangular, and circular solar sail quality membranes were suspended horizontally an supported using two different boundary conditions. These basic configurations enabled the numerical models of the membranes to be fairly simple. Results from the numerical models correlated well to the experimental data. The methods and techniques used to create and validate the models are intended to demonstrate the potential of these experimental and numerical techniques for use in the creation of larger test structures, more detailed finite element models, and eventually full-scale solar sails.

\section{Acknowledgements}

The authors would like to thank Richard Pappa and Jessica Woods-Vedeler of NASA Langley Research Center and Vaughn Behun of Swales Aerospace for their materials, hardware, and testing support. A portion of this work was performed under a Graduate Research Scholar Assistantship provided by NASA Langley through George Washington University.

\section{References}

${ }^{1}$ Spieth, D. and Zubrin, R., "Ultra-Thin Solar Sails for Interstellar Travel - Phase I Final Report," NASA Institute for Advanced Concepts, Pioneer Astronautics Inc., December 1999. 2000.

${ }^{2}$ West, J. L., and Derbes, B., "Solar Sail Vehicle System Design for the GEOStorm Warning Mission," AIAA-2000-5326,

${ }^{3}$ McInnes, C. R., “Solar Sail Mission Opportunities,” Royal Astronomical Society Discussion Meeting,10 May 2002.

${ }^{4}$ McInnes, C. R., Solar Sailing - Technology, Dynamics and Mission Applications, Praxis Publishing Ltd, Chichester, UK, 1999.

${ }^{5}$ Leifer, J., and Belvin, W. K., "Prediction of Wrinkle Amplitudes in Thin Film Membranes Using Finite Element Modeling,” AIAA-2003-1983, April, 2003.

${ }^{6}$ ANSYS Incorporated, Version 6.1 Documentation, Canonsburg, PA 15317

${ }^{7}$ DuPont Corp., Summary of Properties, Kapton Polyimide Film, http://www.dupont.com/kapton/.

${ }^{8}$ Pappa, R. S., Jones, T. W., Black, J. T., Walford, A., Robson, S., and Shortis, M. R., "Photogrammetry Methodology Development for Gossamer Space Structures," Sound and Vibration, Vol. 36, No. 8, August 2002, pp. 12-21.

${ }^{9}$ Pappa, R. S., Black, J. T., Blandino, J. R., Jones, T. W., Danehy, P. M., and Dorrington, A. A., "Dot Projection Photogrammetry and Videogrammetry of Gossamer Space Structures," to appear in the Journal of Spacecraft and Rockets, NASA/TM-2003-212146, February 2003.

${ }^{10}$ Mikhail, E. M., Bethel, J. S., and McGlone, J. C., Introduction to Modern Photogrammetry, John Wiley \& Sons, New York, NY, 2001. 


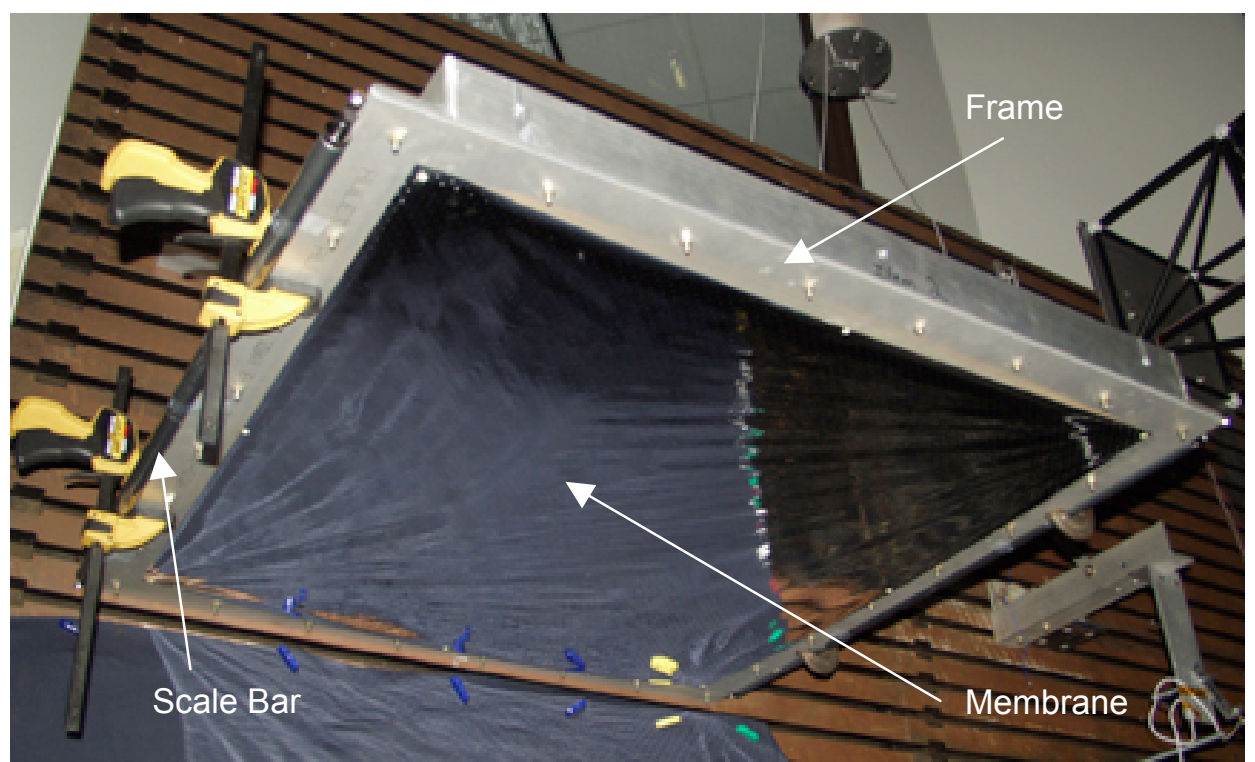

Figure 1 - Horizontally suspended aluminum frame with corner supported membrane

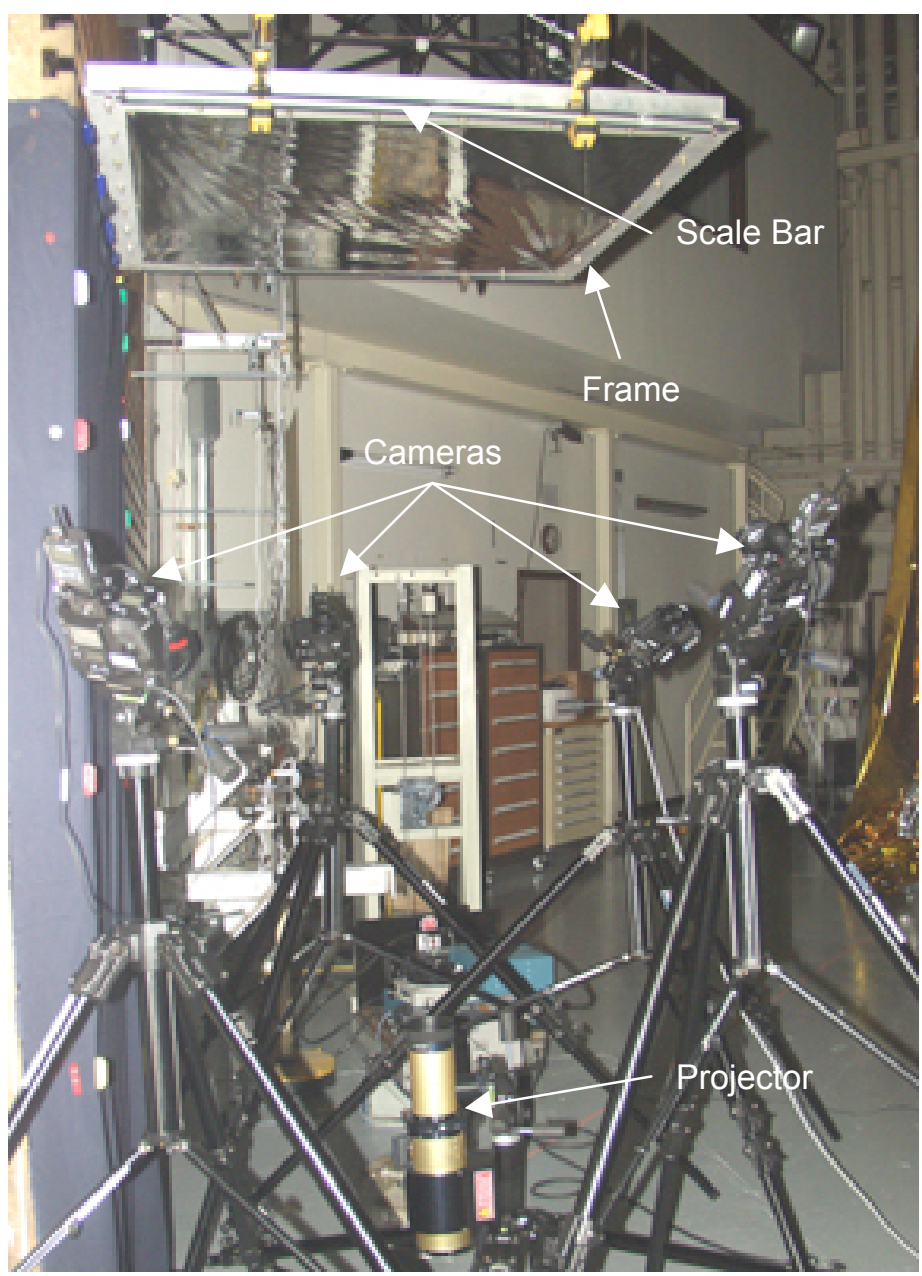

Figure 2 - Test setup 


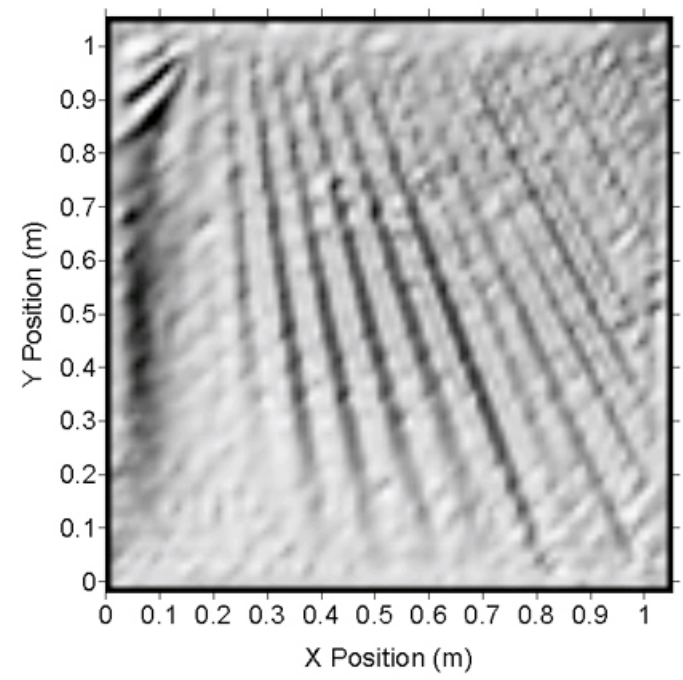

(a) Shaded relief map

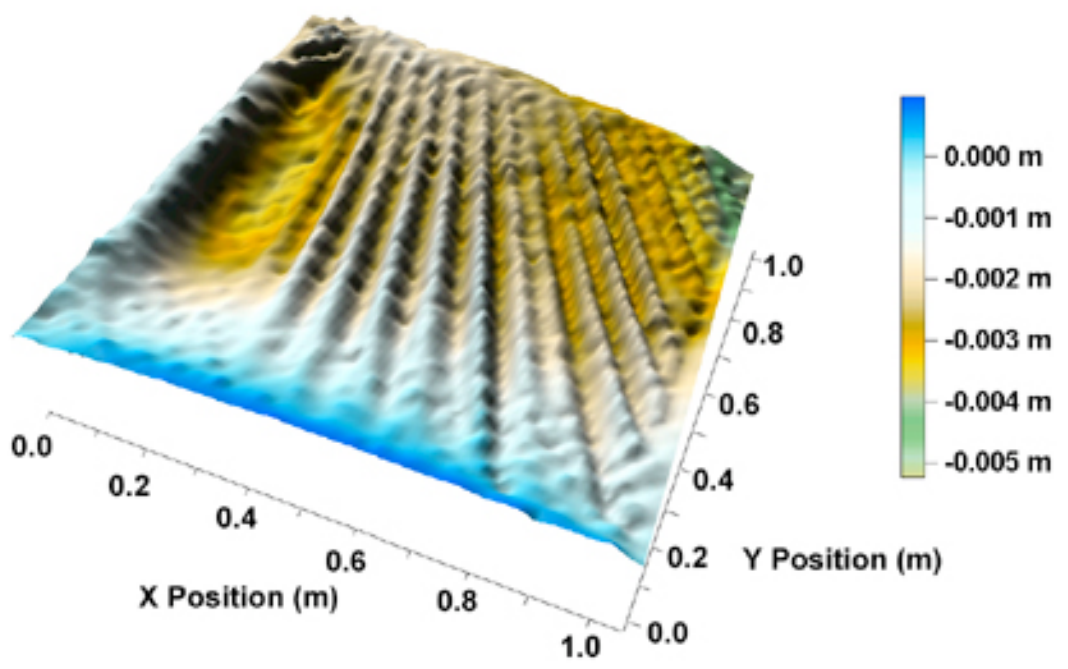

(b) Shaded surface

Figure 3 - Fixed-edge square membrane results

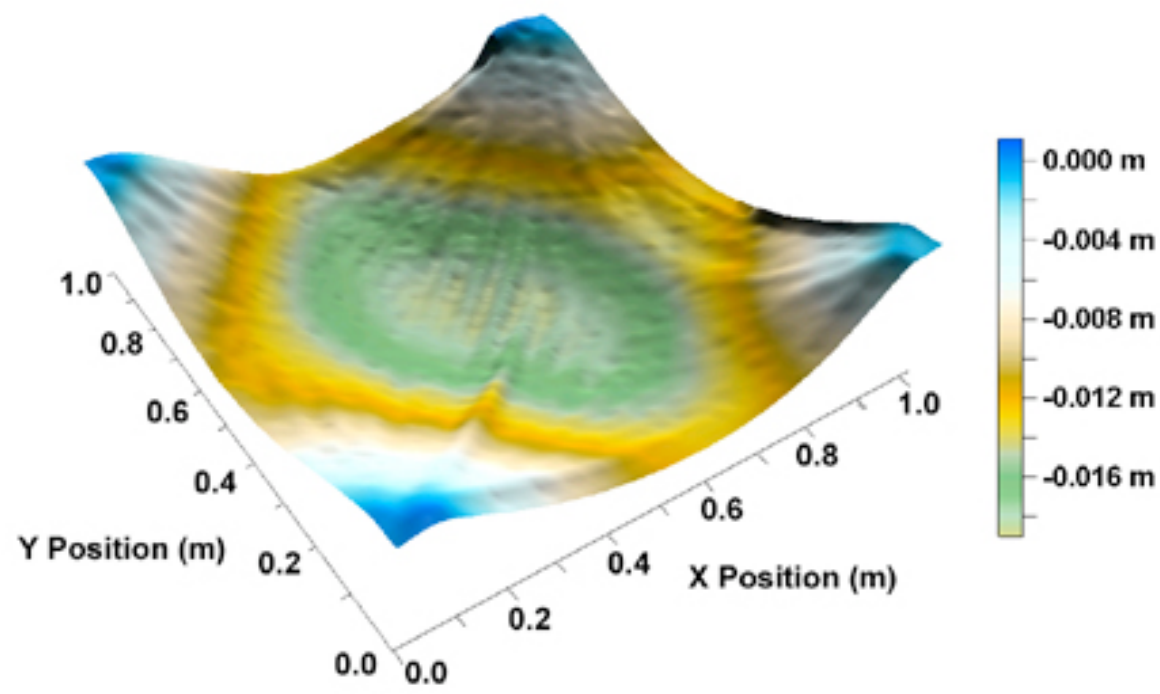

Figure 4 - Corner-attached square membrane results 


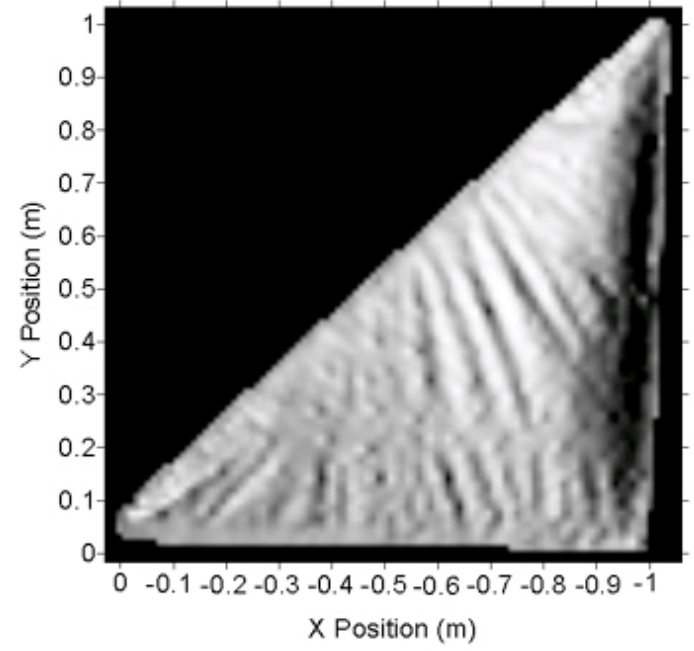

(a) Shaded relief map

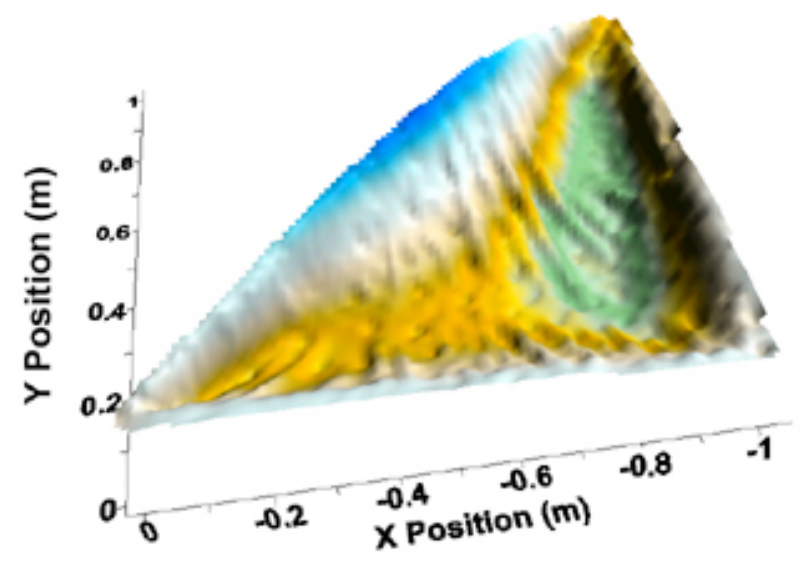

(b) Shaded surface
$0.002 \mathrm{~m}$

$0.001 \mathrm{~m}$

$-0.001 \mathrm{~m}$

$-0.002 \mathrm{~m}$

$-0.003 \mathrm{~m}$

$-0.004 \mathrm{~m}$

Figure 5 - Fixed-edge triangular membrane results

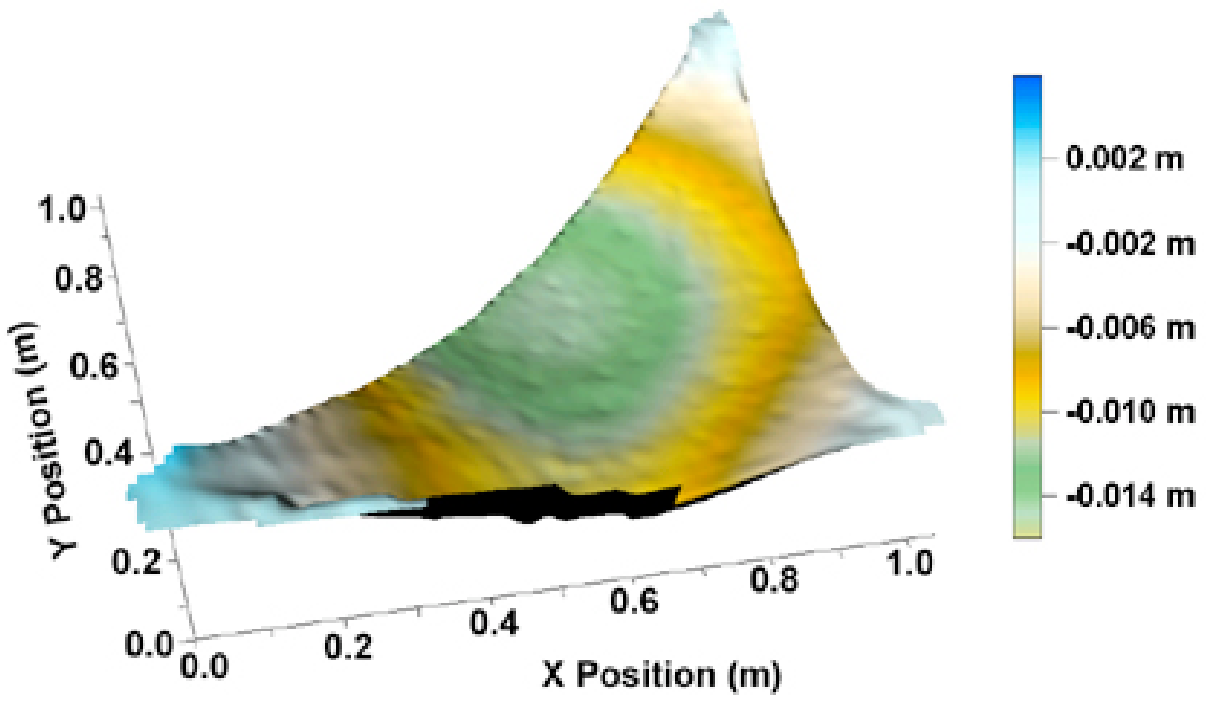

Figure 6 - Corner-attached triangular membrane

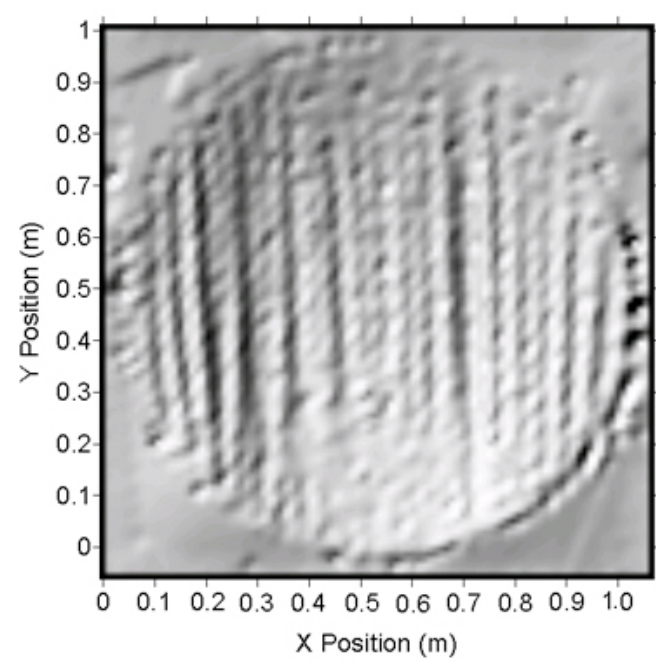

(a) Shaded relief map

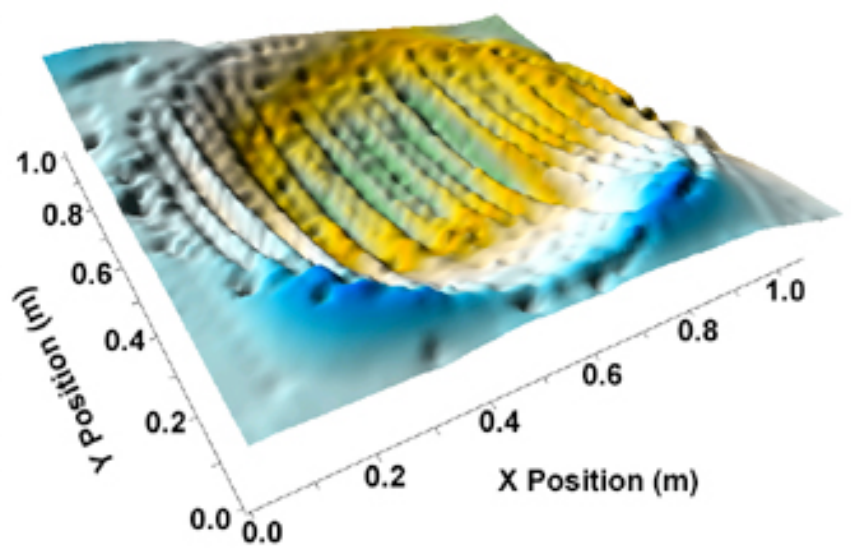

(b) Shaded surface

Figure 7 - Fixed-edge circular membrane results 


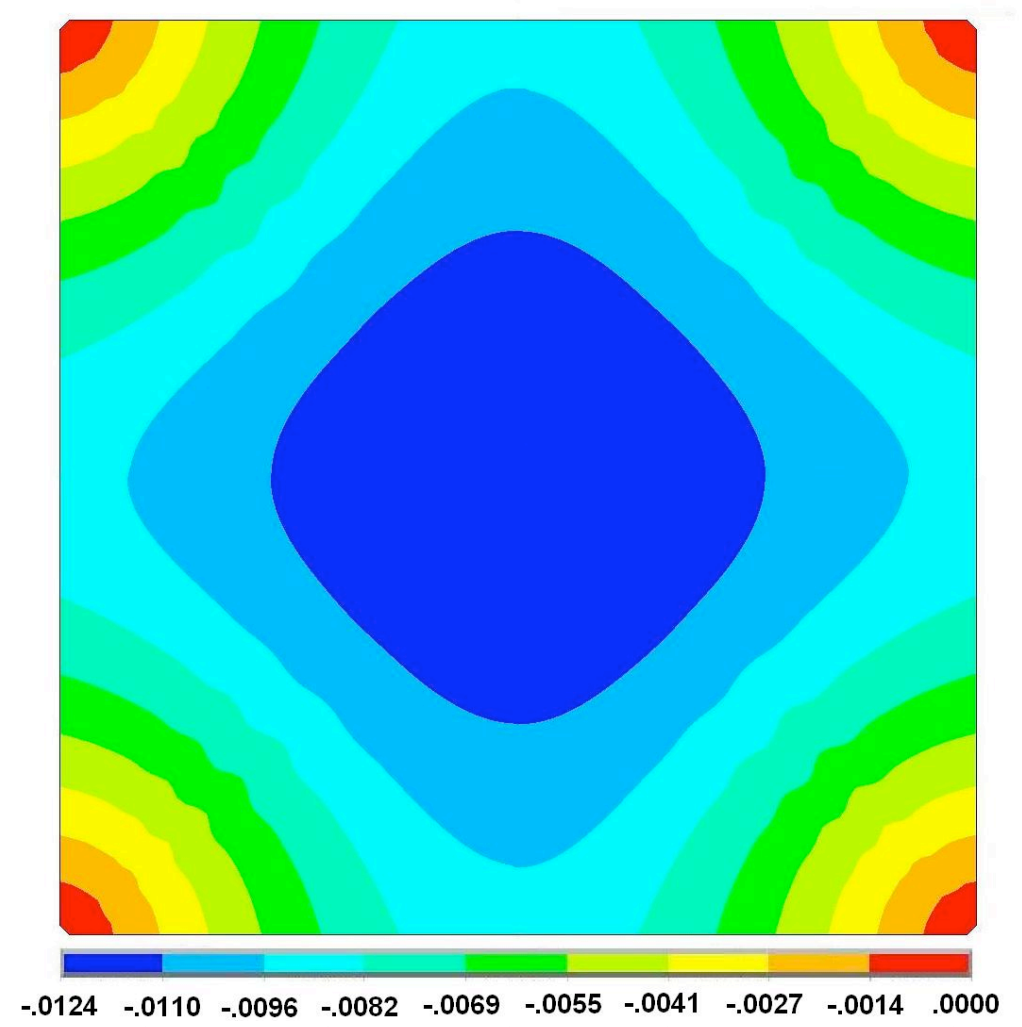

Figure 8 - Corner-supported square membrane finite element results, top-view out of plane deflection in meters

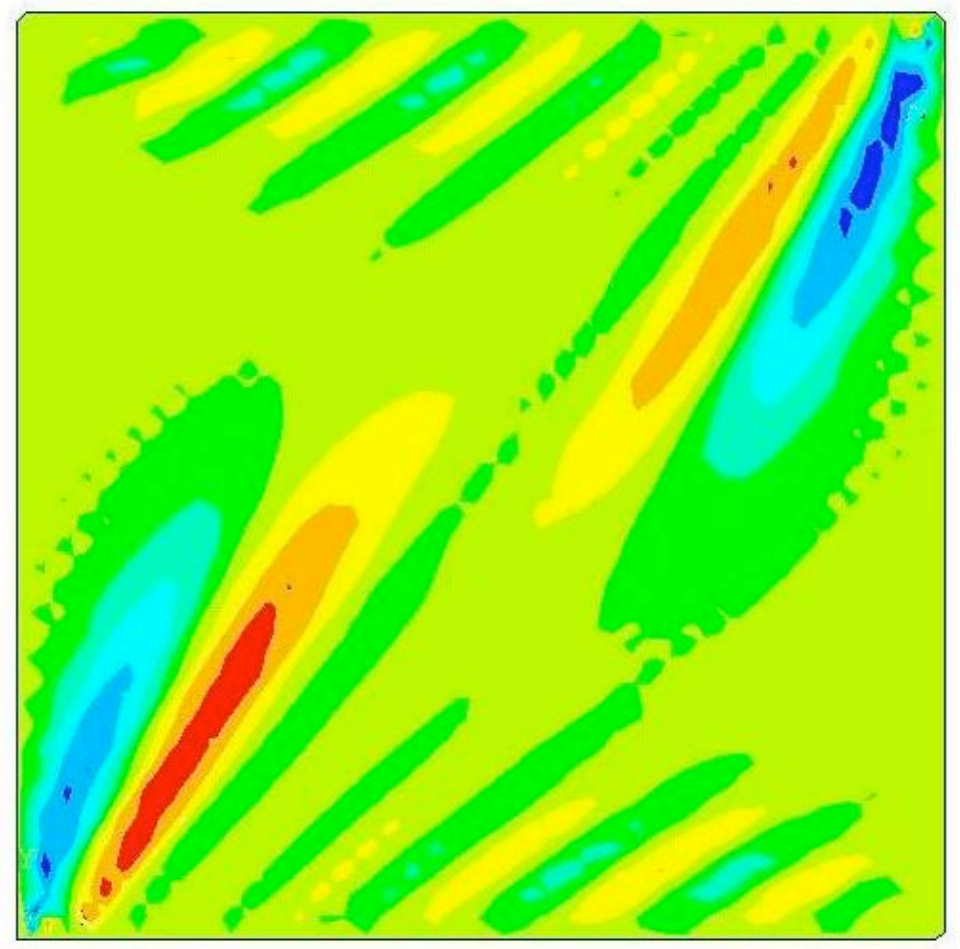

$\begin{array}{llllllllll}-.0037 & -.0030 & -.0023 & -.0016 & -.0009 & -.0002 & .0005 & .0012 & .0019 & .0026\end{array}$

Figure 9 - Fixed-edge square membrane finite element results, top-view out of plane deflection in meters 


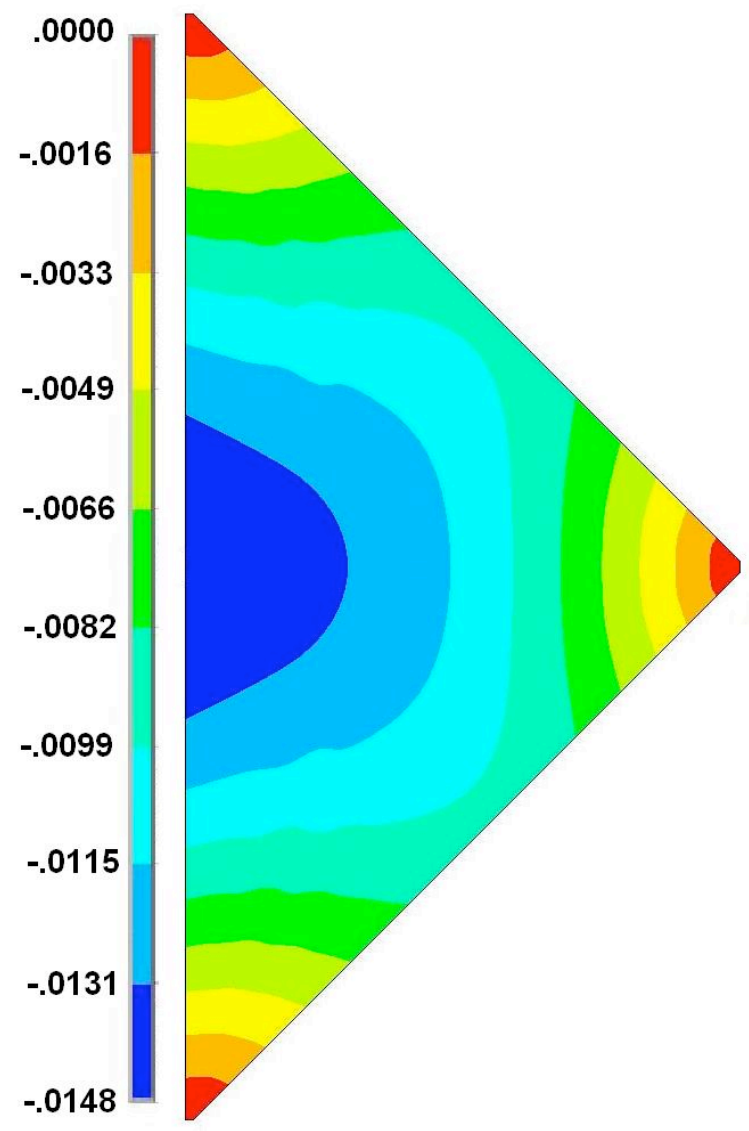

Figure 10 - Corner-supported triangular membrane finite element result, top-view out of plane deflection in meters 